PROCEEDINGS OF THE

AMERICAN MATHEMATICAL SOCIETY

Volume 126, Number 7, July 1998, Pages 2151-2157

S 0002-9939(98)04344-5

\title{
ON SCRAMBLED SETS AND A THEOREM OF KURATOWSKI ON INDEPENDENT SETS
}

\author{
HISAO KATO
}

(Communicated by Mary Rees)

\begin{abstract}
The measure of scrambled sets of interval self-maps $f: I=$ $[0,1] \rightarrow I$ was studied by many authors, including Smítal, Misiurewicz, Bruckner and $\mathrm{Hu}$, and Xiong and Yang. In this note, first we introduce the notion of "*-chaos" which is related to chaos in the sense of Li-Yorke, and we prove a general theorem which is an improvement of a theorem of Kuratowski on independent sets. Second, we apply the result to scrambled sets of higher dimensional cases. In particular, we show that if a map $f: I^{k} \rightarrow I^{k}(k \geq 1)$ of the unit $k$-cube $I^{k}$ is $*$-chaotic on $I^{k}$, then for any $\epsilon>0$ there is a map $g: I^{k} \rightarrow I^{k}$ such that $f$ and $g$ are topologically conjugate, $d(f, g)<\epsilon$ and $g$ has a scrambled set which has Lebesgue measure 1 , and hence if $k \geq 2$, then there is a homeomorphism $f: I^{k} \rightarrow I^{k}$ with a scrambled set $S$ satisfying that $S$ is an $F_{\sigma}$-set in $I^{k}$ and $S$ has Lebesgue measure 1.
\end{abstract}

\section{INTRODUCTION}

All spaces considered in this note are assumed to be separable and complete metric spaces. Maps are continuous functions. By a compactum we mean a compact metric space.

Let $f: X \rightarrow X$ be a map of a compactum $X$ with metric $d$. A subset $S$ of $X$ is a scrambled set of $f$ if there is a positive number $\tau>0$ such that for any $x, y \in S$ with $x \neq y$,

1. $\limsup _{n \rightarrow \infty} d\left(f^{n}(x), f^{n}(y)\right)>\tau$,

2. $\liminf _{n \rightarrow \infty} d\left(f^{n}(x), f^{n}(y)\right)=0$.

If there is an uncountable scrambled set $S$ of $f$, then we say that $f$ is chaotic in the sense of Li-Yorke. In the original paper [6] of Li and Yorke, there was the following one more condition: for any $x \in S$ and any periodic point $p \in X$, $\lim \sup _{n \rightarrow \infty} d\left(f^{n}(x), f^{n}(p)\right)>0$. But it is known that this condition is unnecessary.

In [6], it was proved that if a map $f: I=[0,1] \rightarrow I$ has a periodic point with period 3, then $f$ is chaotic in the sense of Li-Yorke. The measure of scrambled sets of interval self-maps was studied by many authors, including Smítal [11], [12], Misiurewicz [7], Bruckner and Hu [1], and Xiong and Yang [13].

In this note, we prove a general theorem which is an improvement of a theorem [4] of Kuratowski on independent sets, and we apply this result to scrambled sets of higher dimensional cases.

Received by the editors August 29, 1996 and, in revised form, December 20, 1996.

1991 Mathematics Subject Classification. Primary 54H20, 26 A18.

Key words and phrases. Scrambled set, independent set, Cantor set, flat, Lebesgue measure.

(C)1998 American Mathematical Society 
Let $A$ be a closed subset of a compactum $X$. A map $f: X \rightarrow X$ is $*$-chaotic on $A$ (in the sense of Li-Yorke) if the following conditions are satisfied:

1. there is $\tau>0$ such that if $U$ and $V$ are any nonempty open subsets of $A$ with $U \cap V=\emptyset$ and $N$ is any natural number, then there is a natural number $n \geq N$ such that $d\left(f^{n}(x), f^{n}(y)\right)>\tau$ for some $x \in U, y \in V$, and

2. for any nonempty open subsets $U, V$ of $A$ and any $\epsilon>0$ there is a natural number $n \geq 0$ such that $d\left(f^{n}(x), f^{n}(y)\right)<\epsilon$ for some $x \in U, y \in V$.

If $f: X \rightarrow X$ is $*$-chaotic on the total space $X$, we say that $f: X \rightarrow X$ is everywhere $*$-chaotic.

Note that if $S$ is a scrambled set of $f$, then $f$ is $*$-chaotic on $\mathrm{Cl}(S)$. A map $f: X \rightarrow X$ of a compactum $X$ has sensitive dependence on initial conditions on a closed subset $A$ of $X$ if there is $\tau>0$ such that if $x \in A$ and $U$ is any neighborhood of $x$ in $A$, then there is $y \in U$ such that $d\left(f^{n}(x), f^{n}(y)\right)>\tau$ for some $n$. Suppose that $A$ is a closed subset of $X$ and $A$ has no isolated point (=A is perfect). Then $f$ is $*$-chaotic on $A$ if and only if $f$ has sensitive dependence on initial conditions on $A$ and the above condition 2 is satisfied.

In the definition of chaos in the sense of Li-Yorke, we only assume the condition that there exists an uncountable scrambled set $S$ of $f: X \rightarrow X$, and in general, $S$ is an arbitrary subset of $X$. Note that if $S$ is an uncountable set, then $\operatorname{Cl}(S)$ contains a Cantor set by the Cantor-Bendixon theorem (e.g., see [5, p. 253]).

The author would like to thank the referee for helpful comments.

\section{INDEPENDENT SETS AND SCRAMBLED SETS}

In this section, first, we prove the following general theorem which is an improvement of a theorem of Kuratowski on independent sets (see [4]). Let $X$ be a space and $R$ be any subset of $X^{m}(m \geq 2)$. A subset $F \subset X$ is said to be independent in $R$ if for any different $m$ points $x_{1}, \cdots, x_{m}$ of $F$ (i.e., $x_{i} \neq x_{j}$ for $i \neq j$ ), we have $\left(x_{1}, x_{2}, \cdots, x_{m}\right) \in X^{m}-R$. For a subset $R \subset X^{m}$, consider the following property:

$\left.{ }^{*}\right)$ If $U_{1}, \cdots, U_{m}$ are mutually disjoint nonempty sets of $X$, there are nonempty open sets $V_{1}, \cdots, V_{m}$ of $X$ such that $V_{i} \subset U_{i}$ for each $i$ and $V_{1} \times \cdots \times V_{m} \subset$ $X^{m}-R$.

Note that if $X$ has no isolated point, $R \subset X^{m}$ has the property (*) if and only if $R$ is nowhere dense (see Lemma 2.2).

A countable union of nowhere dense sets is called a set of the first category.

Theorem 2.1. Suppose that $X$ is a separable and completely metrizable space and $m \geq 2$ is a fixed natural number. Let $R \subset X^{m}$.

1. If $R=\bigcup_{n=1}^{\infty} R_{n}$ and each $R_{n}$ has the property $(*)$, then there is an $F_{\sigma}$-set $S$ of $X$ such that $S$ is independent in $R$, and $\mathrm{Cl}(S)=X$.

2. If $X$ has no isolated point and $R$ is of the first category, then there is a subset $S$ of $X$ such that $S=\bigcup_{n=1}^{\infty} C_{n}$, where $C_{n}$ are Cantor sets in $X, S$ is independent in $R$, and $\mathrm{Cl}(S)=X$.

To prove this theorem, we need the following lemma by Kuratowski [4, Lemma, p. 66].

Lemma 2.2. Let $X$ be an arbitrary space and let $G$ be an open dense set in the space $X^{m}(m \geq 1)$. Let $H_{1}, \cdots, H_{n}$, where $n \geq m$, be open non-empty sets in $X$. 
Then there exists a system of nonempty open sets $U_{1}, \cdots, U_{n}$ such that $U_{i} \subset H_{i}$ for $1 \leq i \leq n$, and

$$
U_{i_{1}} \times \cdots \times U_{i_{m}} \subset G,
$$

whenever $i_{1}, \cdots, i_{m}$ are different $m$ elements of $\{1,2, \cdots, n\}$.

Proof of Theorem 2.1. We prove the second case. The first case can be similarly proved.

We assume that $X$ has no isolated points. Also, we may assume that $R=$ $\bigcup_{k=1}^{\infty} R_{k}$, where $R_{k}$ is nowhere dense and a closed subset of $X^{m}$ for each $k$. Let $\left\{U_{i}\right\}_{i=1}^{\infty}$ be a countable open base of $X$. Let $i=1$. By the above lemma, we can choose a family

$$
\mathcal{V}_{1}=\left\{V_{1,1}, V_{1,2}, \cdots, V_{1, n_{1}}\right\}
$$

of mutually disjoint nonempty closed subsets of $X$ such that $\operatorname{Int}\left(V_{1, j}\right) \neq \emptyset$ for each $j,\left(\bigcup \mathcal{V}_{1}\right) \cap U_{1} \neq \emptyset$, and for any different $m$ elements $V_{1, j_{1}}, \cdots, V_{1, j_{m}}$ of $\mathcal{V}_{1}$,

$$
\left(V_{1, j_{1}} \times \cdots \times V_{1, j_{m}}\right) \cap\left(R_{1}\right)=\emptyset,
$$

where $\bigcup \mathcal{V}_{1}=\bigcup\left\{V \mid V \in \mathcal{V}_{1}\right\}$.

Inductively, by the above lemma we can choose a sequence

$$
\mathcal{V}_{i}=\left\{V_{i, 1}, \cdots, V_{i, n_{i}}\right\}(i \geq 1)
$$

of families of mutually disjoint nonempty closed subsets of $X$ such that

1. $\operatorname{Int}\left(V_{i, j}\right) \neq \emptyset$ and $\operatorname{mesh}\left(\mathcal{V}_{i}\right)<1 / i$,

2. for each $V_{i, j}$, there are at least two elements $V_{i+1, p}, V_{i+1, q}(p \neq q)$ of $\mathcal{V}_{i+1}$ such that $V_{i+1, p} \cup V_{i+1, q} \subset \operatorname{Int}\left(V_{i, j}\right)$,

3. $U_{i} \cap\left(\bigcup \mathcal{V}_{i}\right) \neq \emptyset$, and

4. for any different $m$ elements $V_{i, j_{1}}, \cdots, V_{i, j_{m}}$ of $\mathcal{V}_{i}$,

$$
\left(V_{i, j_{1}} \times \cdots \times V_{i, j_{m}}\right) \cap\left(\bigcup_{k=1}^{i} R_{k}\right)=\emptyset .
$$

For each $i$, by induction we can obtain the sequence $\{i, j(j=i, i+1, i+2, \cdots)$ of subfamilies of $\mathcal{V}_{j}$ as follows:

$\mathcal{F}_{i, i}=\mathcal{V}_{i}, \quad \mathcal{F}_{i, j+1}=\left\{V \in \mathcal{V}_{j+1} \mid V\right.$ is contained in some element of $\left.\mathcal{F}_{i, j}\right\}(j \geq i)$.

Put $C_{i}=\bigcap_{j=i}^{\infty}\left(\bigcup \mathcal{F}_{i, j}\right)$. Then we see that $C_{i}$ is a Cantor set and $\left\{C_{i}\right\}_{i=1}^{\infty}$ is increasing. Then by the condition $4, S=\bigcup_{i=1}^{\infty} C_{i}$ is independent in $R=\bigcup_{k=1}^{\infty} R_{k}$ and by the condition $3, S$ is dense in $X$. This completes the proof.

Let $I=[0,1]$ be the unit interval and $I^{k}(k \geq 1)$ the unit $k$-cube. A space homeomorphic to $I^{k}$ is a $k$-cell. A $k$-cell $B$ in $I^{k}-\partial I^{k}$ is flat if there is a homeomorphism $h: I^{k} \rightarrow I^{k}$ such that $h(B)=J^{k}$, where $J=[1 / 3,2 / 3] \subset I$. A 0-dimensional compactum $D$ in $I^{k}-\partial I^{k}$ is flat in $I^{k}$ if for any neighborhood $V$ of $D$ in $I^{k}$, there is a neighborhood $U$ of $D$ in $I^{k}$ such that $U \subset V$ and $U=B_{1} \cup \cdots \cup B_{p}$, where $B_{i}(i=1,2, \cdots, p)$ are mutually disjoint $k$-cells. Then we may assume that $B_{i}$ is flat in $I^{k}$ for each $i$, because we can choose a $k$-cell $B^{\prime}$ in $\operatorname{Int}\left(B_{i}\right)$ such that $\partial B^{\prime}$ is locally flat and hence $B^{\prime}$ is flat by Generalized Schoenflies Theorem (e.g. see [10, p. 48]). Note that if $C$ and $C^{\prime}$ are flat Cantor sets in $I^{k}$ and $k \geq 2$, then any homeomorphism $f: C \cup \partial I^{k} \rightarrow C^{\prime} \cup \partial I^{k}$ can be extended to a homeomorphism $F: I^{k} \rightarrow I^{k}$ (e.g., see the proof of $[8$, p. 93, Theorem 7]). Also, note that any 
closed subset of a flat 0-dimensional compactum is also flat. It is well known that if $k \leq 2$, any Cantor set in $I^{k}-\partial I^{k}$ is flat, but if $k \geq 3$, there are Cantor sets in $I^{k}-\partial I^{k}$ which are not flat (see $[8$, p. 127]).

Proposition 2.3. Suppose that $X$ is the unit $k$-cube $I^{k}(k \geq 1)$. Let $m \geq 2$ be a fixed natural number and let $R \subset X^{m}$ be of the first category in $X^{m}$. Then there is a subset $S$ of $X$ such that $S=\bigcup_{n=1}^{\infty} C_{n}$, where $C_{n}$ are flat Cantor sets in the unit $k$-cube $X, S$ is independent in $R$, and $C l(S)=X$.

Proof. In the proof of the above theorem, we may assume that each $V_{i, j}$ is a $k$-cell (see Lemma 2.2).

Theorem 2.4. If $f: X \rightarrow X$ is a map of a compactum $X$ and $f$ is $*$-chaotic on a closed set $A$, then there is an $F_{\sigma}$-set $S \subset A$ such that $S$ is a scrambled set of $f$ and $C l(S)=A$. If $A$ has no isolated points, we can choose $S$ such that $S$ is a countable union of Cantor sets $C_{n}$. Moreover if $X=I^{k}$, then the Cantor sets $C_{n}$ can be chosen as flat Cantor sets in $X$.

Proof. Suppose that $\tau$ is a positive number as in the definition of scrambled set $S$. Consider the following sets:

$$
\begin{aligned}
& R_{1}=\left\{(x, y) \in A^{2} \mid \limsup _{i \rightarrow \infty} d\left(f^{i}(x), f^{i}(y)\right)<\tau\right\}, \\
& R_{2}=\left\{(x, y) \in A^{2} \mid \liminf _{i \rightarrow \infty} d\left(f^{i}(x), f^{i}(y)\right)>0\right\} .
\end{aligned}
$$

Let $\epsilon_{n}=1 / n(n=1,2, \cdots)$. Then $R_{1}=\bigcup_{n=1}^{\infty} T_{n}$, where

$$
T_{n}=\left\{(x, y) \in A^{2} \mid d\left(f^{i}(x), f^{i}(y)\right) \leq \tau-\epsilon_{n} \text { for every } i \geq n\right\} .
$$

Also, $R_{2}=\bigcup_{n=1}^{\infty} W_{n}$, where

$$
W_{n}=\left\{(x, y) \in A^{2} \mid d\left(f^{i}(x), f^{i}(y)\right) \geq \epsilon_{n} \text { for every } i \geq n\right\} .
$$

Note that $T_{n}, W_{n} \subset A^{2}$ are closed. Since $f$ is *-chaotic on $A$, they have the property $(*)$. By Theorem 2.1 and Proposition 2.3 , we obtain a desired scrambled set $S$.

Corollary 2.5. Let $f: X \rightarrow X$ be a map of a compactum $X$ and $S$ an uncountable scrambled set of $f$. Then there is an $F_{\sigma}$-set $S^{\prime}$ of $X$ such that $S^{\prime}$ is a scrambled set of $f, C l\left(S^{\prime}\right)=C l(S)$, and $S^{\prime}$ contains a Cantor set. Moreover, if $C l(S)$ has no isolated point, $S^{\prime}$ can be chosen so that $S^{\prime}$ is a countable union of Cantor sets $C_{n}$. Moreover if $X=I^{k}$, then the Cantor sets $C_{n}$ can be chosen as flat Cantor sets in $I^{k}$.

Let $\mu$ be the Lebesgue measure on $I^{k}$. Note that there are subsets $E$ of $I^{k}-\partial I^{k}$ with $\mu(E)=1$ which are countable union of flat Cantor sets in $I^{k}$.

Theorem 2.6. Suppose that $E$ is a countable union of flat Cantor sets of $I^{k}(k \geq$ 1) such that $\mu(E)=1$. If $f: I^{k} \rightarrow I^{k}$ is everywhere $*$-chaotic, then for any $\epsilon>0$ there is a map $g: I^{k} \rightarrow I^{k}$ such that $f$ and $g$ are topologically conjugate, $d(f, g)<\epsilon$ and $E$ is a scrambled set of $g$, where $d(f, g)=\sup \{d(f(x), g(x)) \mid x \in X\}$. In particular, $g$ has a scrambled set which has Lebesgue measure 1. 
Proof. First, we assume $k \geq 2$. Note that the space $H(X)$ of all homeomorphisms of a compactum $X$ has a complete metric, i.e., $\rho(f, g)=d(f, g)+d\left(f^{-1}, g^{-1}\right)$. By Theorem 2.4, we can choose a scrambled set $S$ of $f$ such that $S$ is a countable union of Cantor sets which are flat in $I^{k}$, and $S$ is dense in $I^{k}$. Let $\epsilon_{1}>\epsilon_{2}>\cdots$ be a sequence of positive numbers with $\sum_{n=1}^{\infty} \epsilon_{n}<\epsilon$. Since any closed subset of a flat Cantor set is also flat, we can choose mutually disjoint flat Cantor sets $D_{n}(n=1,2, \cdots)$ such that $E=\bigcup_{n=1}^{\infty} D_{n}$. For $n=1$, we choose mutually disjoint $k$-cells $B_{j}(j=1,2, \cdots, p)$ such that $U=\bigcup_{j=1}^{p} B_{j}$ is a neighborhood of $D_{1}$ and $\operatorname{diam}\left(B_{j}\right)<\epsilon_{1}$. Also, since any closed subset of a flat Cantor set is flat, we can choose a flat Cantor set $C_{1} \subset S \cap \operatorname{Int}(U)$ such that $C_{1} \cap B_{j} \neq \emptyset$ for each $j$. Then there is a homeomorphism $h_{1}: I^{k} \rightarrow I^{k}$ such that $h_{1}\left(D_{1}\right)=C_{1} \subset S, h_{1} \mid I^{k}-U=i d$ (see the proof of $\left[8\right.$, p. 93, Theorem 7]). Then $\rho\left(i d, h_{1}\right)<\epsilon_{1}$. Put $D^{\prime}=h_{1}\left(D_{2}\right)$. Note that $D^{\prime}$ is flat. We can choose a neighborhood $V$ of $D^{\prime}$ such that $V \cap h_{1}\left(D_{1}\right)=\emptyset$. By the above argument, we obtain a homeomorphism $h^{\prime}: I^{k} \rightarrow I^{k}$ such that $h^{\prime}\left(D^{\prime}\right) \subset S, h^{\prime} \mid I^{k}-V=i d, \rho\left(i d, h^{\prime}\right)<\epsilon_{2}$. Put $h_{2}=h^{\prime} \cdot h_{1}$.

If we continue this procedure, we obtain a sequence $h_{n}: I^{k} \rightarrow I^{k}$ of homeomorphisms such that

1. $\rho\left(h_{1}, 1\right)<\epsilon_{1}, \rho\left(h_{n}, h_{n+1}\right)<\epsilon_{n}$ for each $n$,

2. $h_{n+1}\left|\left(\bigcup_{j=1}^{n} D_{j}\right)=h_{n}\right|\left(\bigcup_{j=1}^{n} D_{j}\right)$, and $h_{n}\left(D_{n}\right) \subset S$.

Then we obtain a homeomorphism $h=\lim _{n \rightarrow \infty} h_{n}$, since $H(X)$ is complete and the sequence $\left\{h_{n}\right\}_{n=1}^{\infty}$ is a Cauchy sequence. By the condition 2, we see that $h(E) \subset S$. Put $g=h^{-1} \cdot f \cdot h: I^{k} \rightarrow I^{k}$. Then $g$ is a desired map. The case $k=1$ is similarly proved by using Theorem 2.4 and [2]. This completes the proof.

Remark 2.7. For the proof of a weak version of Theorem 2.6, we can use the following theorem [9, Theorem 9] by Oxtoby and Ulam: for any subset $B$ of any $k$-cube $R(k \geq 1)$, there is a homeomorphism $h: R \rightarrow R$ with $h \mid \partial R=i d$ such that $\mu(h(B))=0$ if and only if $R-B$ contains a sequence of perfect sets whose union is dense in $R$. In fact, for any $\delta>0$, we decompose $I^{k}$ into $k$-cubes $R_{1}, \cdots, R_{p}$ such that $\operatorname{Int}\left(R_{i}\right) \cap \operatorname{Int}\left(R_{j}\right)=\emptyset(i \neq j), \operatorname{diam}\left(R_{i}\right)<\delta$, and $I^{k}=R_{1} \cup \ldots \cup R_{p}$. If $S$ is a scrambled set of $f$ such that $S$ is dense in $I^{k}$ and $S$ is a countable union of Cantor sets, then for each $R_{i}$ there is a homeomorphism $h_{i}: R_{i} \rightarrow R_{i}$ such that $h_{i} \mid \partial R_{i}=i d, \mu\left(h_{i}\left(R_{i}-S\right)\right)=0$. Define a map $h: I^{k} \rightarrow I^{k}$ by $h \mid R_{i}=h_{i}$. Put $S^{\prime}=h(S)$ and $g=h \cdot f \cdot h^{-1}$. Then $\mu\left(S^{\prime}\right)=1$. If we choose a sufficiently small positive number $\delta$, then $g$ has a scrambled set $S^{\prime}$ which has Lebesgue measure 1, and $d(f, g)<\epsilon$.

By [3, Corollary 3.3], every compact connected $k$-manifold $(k \geq 2)$ admits an everywhere $*$-chaotic homeomorphism. Hence we obtain

Corollary 2.8. There is a homeomorphism $f: I^{k} \rightarrow I^{k}(k \geq 2)$ such that there is a scrambled set $S$ of $f$ with $\mu(S)=1$.

Remark 2.9. There is no homeomorphism $f: I \rightarrow I$ with a nonempty scrambled set.

By the similar proof of Theorem 2.6, we obtain the following.

Theorem 2.10. Let $f: I^{k} \rightarrow I^{k}$ be a map of the unit $k$-cube $I^{k}$ and suppose that there is an uncountable scramble set $S$ of $f$ with $S \subset I^{k}-\partial I^{k}$. Then for any $\epsilon>0$ there is a map $g: I^{k} \rightarrow I^{k}$ such that $d(f, g)<\epsilon, g$ is topologically conjugate to 
$f$, and there is a scrambled set $S^{\prime}$ of $g$ such that $S^{\prime}$ is a Cantor set and $S^{\prime}$ has a positive Lebesgue measure.

Let $f: X \rightarrow X$ be a map of a compactum $X$. A point $x \in X$ is recurrent if for any neighborhood $U$ of $x$ in $X$, there is a natural number $N>0$ such that $f^{N}(x) \in U$. A point $x \in X$ is nonwandering if for any neighborhood $U$ of $x$ in $X$, there is a natural number $N>0$ such that $f^{N}(U) \cap U \neq \emptyset$. If there is a point of $X$ whose orbit is dense, then $f$ is called transitive. It is well known that the set $\Omega(f)$ of all nonwandering points of $f$ is closed, and the set $R(f)$ of all recurrent points of $f$ is $G_{\delta}$. Put $T(f)=\{x \in X \mid$ the orbit of $x$ is dense in $X\}$. Then $T(f)$ is $G_{\delta}$-dense if $f$ is transitive. Note that if $X=\Omega(f)$, then $R(f)$ is dense in $X$. Note that, in general, if a set $F$ is of the first category in $X$, then $(F \times X) \cup(X \times F)$ is of the first category in $X^{2}$. Hence by the same argument as above, we can add the following restriction to scrambled sets.

Corollary 2.11. Let $f: I^{k} \rightarrow I^{k}$ be an everywhere $*$-chaotic map.

1. If $f$ is transitive, then for any $\epsilon>0$ there is a map $g: I^{k} \rightarrow I^{k}$ such that $f$ and $g$ are topologically conjugate, $d(f, g)<\epsilon$ and there is a scrambled set $S \subset T(g)$ of $g$ which has Lebesgue measure 1.

2. If $\Omega(f)=X$, then for any $\epsilon>0$ there is a map $g: I^{k} \rightarrow I^{k}$ such that $f$ and $g$ are topologically conjugate, $d(f, g)<\epsilon$ and there is a scrambled set $S \subset R(g)$ of $g$ which has Lebesgue measure 1.

A map $f: X \rightarrow X$ is mixing if for nonempty open sets $U, V$ of $X$, there is a natural number $N$ such that if $n>N$, then $f^{n}(U) \cap V \neq \emptyset$.

Corollary 2.12 (cf. [13]). Let $f: I^{k} \rightarrow I^{k}$ be a mixing map. Then for any $\epsilon>0$ there is a map $g: I^{k} \rightarrow I^{k}$ such that $f$ and $g$ are topologically conjugate, $d(f, g)<\epsilon$ and there is a scrambled set $S \subset T(g)$ of $g$ which has Lebesgue measure 1.

Remark 2.13 (cf. [4, Applications]). 1. There is a Vitali set in the space $E$ of reals containing a countable union $S$ of Cantor sets such that $S$ is dense in E.

2. If $X$ is an indecomposable continuum, then $X$ contains a subset $S$ such that $S$ is a countable union of Cantor sets, $S$ is dense in $X$, and no two of points of $S$ belong to the same composant of $X$.

\section{REFERENCES}

1. B A. M. Bruckner and T. Hu, On scrambled sets for chaotic functions, Trans. Amer. Math. Soc. 301 (1987), 289-297. MR 88f:26003

2. W. J. Gorman III, The homeomorphic transformation of c-sets into d-sets, Proc. Amer. Math. Soc. 17 (1966), 825-830. MR 34:7734

3. H. Kato, Attractors and everywhere chaotic homeomorphisms in the sense of Li-Yorke on manifolds and $k$-dimensional Menger manifolds, Topology Appl. 72 (1996), 1-17.

4. Ku K. Kuratowski, Applications of Baire-category method to the problem of independent sets, Fund. Math. 81 (1973), 65-72. MR 49:3855

5. K. Kuratowski, Topology I, PWN, Warsaw, 1966. MR 36:840

6. T. Y. Li and J. A. Yorke, Period three implies chaos, Amer. Math. Monthly, 82 (1975), 985-992. MR 52:5898

7. M. Misiurewicz, Chaos almost everywhere, Lecture Notes in Math. 1163 (1985), 125-130. MR 87e:58152

8. E. E. Moise, Geometric topology in dimension 2 and 3, Springer, 1977. MR 58:7631

9. J. C. Oxtoby and S. M. Ulam, Measure-preserving homeomorphisms and metrical transitivity, Ann. of Math. 42 (1941), 873-920. MR 3:211b 
10. T. B. Rushing, Topological embeddings, Academic Press, New York, 1973. MR 50:1247

11. J. Smítal, A chaotic function with some extremal properties, Proc. Amer. Math. Soc. 87 (1983), 54-56. MR 84h:26008

12. J. Smítal, A chaotic function with a scrambled set of positive Lebesgue measure, Proc. Amer. Math. Soc. 92 (1984), 50-54. MR 86b:26009b

13. J. Xiong and Z. Yang, Chaos caused by a topologically mixing map, World Scientific, Advanced Series in Dynamical Systems Vol 9, 550-572. MR 93c:58153

Institute of Mathematics, University of Tsukuba, Ibaraki, 305 Japan

E-mail address: hisakato@sakura.cc.tsukuba.ac.jp 